\title{
BIRDS IN AND AROUND SRI VENKATESWARA WILDLIFE SANCTUARY, ANDHRA PRADESH
}

\author{
B. Anand Mohan \\ Curator, Indira Gandhi Zoological Park, Visakhapatnam, Andhra Pradesh, India
}

\begin{abstract}
A study of the bird fauna of Sri Venkateshwara Wildlife Sanctuary, Tirupathi, Andhra Pradesh revealed a total of 178 species. The study was conducted over three years in different forest types such as wooded forests, dry deciduous forests, wet lands, open scrub jungle and adjoining agricultural fields.
\end{abstract}

\author{
Keywords \\ Birds, inventory, Sri Venkateshwara Wildlife \\ Sanctuary, occurrence, habitat
}

\section{Introduction}

Very little information is available on the avifaunal resources of Andhra Pradesh through the work done by Whistler and Kinner (1930-37) on the Vernay Scientific survey of the Eastern Ghats and the Hyderabad State Ornithological Survey (Ali, 1933-34). Later works were done by Abdulali (1945-53), S.A. Hussain (1976) Shahid Ali (1976-83), K.S.R. Krishanam Raju (1971-85), N. Majumder (1978), Trevor Price (1976-83), S.D. Ripley (1975-88), B.M. Beehler (1985) etc. They are mostly restricted to the northern Eastern Ghats in Andhra Pradesh and a few other patches in the state. In its earlier study, the Bombay Natural History Society (BNHS) had field stations at Mamandur (Chittoor District), Guvvalacheruvu and Ontimetta (Cuddapah District). The BNHS monitored the field stations from time to time for avifaunal resources. No specific complete checklist of birds of Sri Venkateswara Wildlife Sanctuary was prepared by any agency except, the reference of some common birds of the districts in the District Forest Working Plans and checklist of birds in certain divisions. In the present study, the checklist and the status of avifauna of Sri Venkateswara Wildlife Sanctuary and its adjoining areas is prepared.

\section{Study area}

The area of study was 525 sq.km. +50 sq.km. which included Sri

Received on 31 March 1999 Accepted on 14 August 2000
Venkateswara Wildlife Sanctuary, Tirupathi and its adjoins. The Sanctuary lies between $13^{\circ} 38^{\prime} \mathrm{N}$ and $13^{\circ} 55^{\prime} \mathrm{N}$ latitude; $79^{\circ} 11^{\prime} 20^{\prime \prime} \mathrm{E}$ and $79^{\circ} 29^{\prime} 10^{\prime \prime}$ longitude. The elevation of the area ranges from 150 to $1129 \mathrm{~m}$. Terrain is undulating. Average rainfall $900 \mathrm{~mm}$. Bulk of rainfall is from the North-East monsoon in addition to the scanty South-West monsoon. The area has 1500 vascular plant species belonging to 174 families, of which nine are endemic. Sri Venkateswara Wildlife Sanctuary is situated in the southern Eastern Ghats of Andhra Pradesh. The Sanctuary was established in 1985. Parts of the Sanctuary is in Chittoor and Cuddapah districts.

\section{Materials and methods}

From August 1991 to August 1994, the following field stations were monitored for avifauna. 1. Tirumala (Well wooded forest); 2. Talakona (Well wooded forest); 3. Chamala (Dry deciduous forest); 4. Mamandur (Dry deciduous forest. This also includes Anjancyapuram and Mamandur Village tanks, which come under wet lands); 5. Balapally (Dry deciduous forest. This also includes Settigunta Village tank, which comes under wet lands); 6. Tirupathi (Open scrub forest); and 7. Srivarimettu (Open scrub forest).

For the purpose of this study, adjoining wetlands and village fields approximately to an extent of another $50 \mathrm{sq} . \mathrm{km}$. was also included in the study area, as the ecological boundaries of certain bird species were overlapping with the adjoining areas. Thus the study area was $575 \mathrm{sq} . \mathrm{km}$. in all. For the purpose of the study, the vegetation and the approximate extent of the area was marked as well wooded (WW 9\%), dry deciduous (DD 63\%), open scrub (OS 20\%) and wet land area (WL 8\%).

Based on the frequency of sightings in the field visits, the following four categories were made on their occurrence.

Common: Observed through out the study in fairly good numbers and abundant in a particular locality. In general, if the percentage of recordings were more than 50 times they were grouped in this category. 
Occasional: Sightings were less than 50 per cent and more than 20 per cent.

Uncommon: Found in small numbers and with less frequency. Sightings were less than 20 per cent and more than five per cent. Mostly the seasonal migratory birds of the wet lands belong to this category.

Rare: Seen in very small numbers and their frequency of sightings were less than five per cent.

\section{Results and Discussion}

During the study period, 178 species of birds were recorded in the area. Taking account of the frequency of habitat preference, each species was assigned to one of the categories. For the purpose of assigning habitat preference, certain species like Blue Rock Pigeon, which occurs in the rocky ledges of the hills, were combined with the well wooded forests and species like Yellow Wagtail, Grey Wagtail, Large Pied Wagtail were assigned to wet land habitat, since they prefer stream banks and other wet land/marshy area associated species like Baya were also assigned in the wet land category according to their habitat

Table 1. The occurrence and habitat preference of birds in Sri Venkateswara Wildlife Sanctuary

\begin{tabular}{l|llll|l}
\hline Occurrence & \multicolumn{4}{|c|}{ Habitat preference } & \multirow{2}{*}{ Total } \\
\cline { 2 - 5 } & $\begin{array}{l}\text { Well } \\
\text { Wooded }\end{array}$ & $\begin{array}{l}\text { Dry } \\
\text { Deciduous Scrub }\end{array}$ & $\begin{array}{l}\text { Open } \\
\text { Land }\end{array}$ & \\
\hline Common & 5 & 13 & 30 & 6 & 54 \\
Occasional & 1 & 5 & 8 & 6 & 20 \\
Uncommon & 11 & 16 & 27 & 37 & 91 \\
Rare & 3 & 3 & 1 & 6 & 13 \\
Total & 20 & 37 & 66 & 55 & 178 \\
\hline
\end{tabular}

preference. The details of categorization of the total recorded bird species of the study area, according to their occurrence and habitat preference are given in Table 1.

The present study recorded 178 species of birds, belonging to 49 families. Nearly 40 species of wet land birds are migratory winter visitors. The main season for winter visitors is from September to February. There are 15 species of raptors. Osprey is a winter visitor found in the area from September to February. Yellow-browed Bulbul which is found in the Western Ghats is found rarely in the well wooded semi evergreen riparian forests in the study area. Details of occurrence and habitat preference of each species are given in Table 2. The scientific names and taxonomic classification of birds listed in table 1 follow Inskipp et al. (1996). The common names follows Manakadan et al. (1998).

\section{Acknowledgements}

The author is thankful to the Andhra Pradesh Forest Department for providing the opportunity to work in the area; to Prof. Rama Murthy, Former Vice-Chancellor, S.V. University, Tirupathi for his encouragement.

\section{References}

Ali, S. (1997). The book of Indian birds. Bombay Natural History Society.

Ali, S. and S.D. Ripley (1983). A pictorial guide to the birds of the Indian subcontinent. Oxford University Press, New Delhi, 177 pp.

Anand Mohan, B. (1991). Check list of birds (114 Nos) of S.V.N.P. and S.V.W.L.S. Forest Department of Andhra Pradesh.

Inskipp, T., N. Lindsey \& W. Duckworth (1996). An Annotated Checklist of the Birds of the Oriental Region. Oriental Bird Club, U.K. Manakadan, R., J.C. Daniel, A.R. Rahmani, M. Inamdar and G. Ugra (1998). Standardized English common names of the birds of the Indian sub-continent - a proposal. Buceros 3(2): 55 pp. Bombay Natural History Society, Mumbai.

Osman, S.M. (1991). Hunters of the air. WWF India.

Taher, S.A. and A. Pittie (1989). A checklist of birds of Andhra Pradesh, Pitta 14: ii

Woodcock, M.W. (1979). Collins Handguide to the birds of the Indian Sub-continent. Collins, London, 176 pp. 
Table 2. Birds in and around Sri Venkateswara Wildlife Sanctuary.

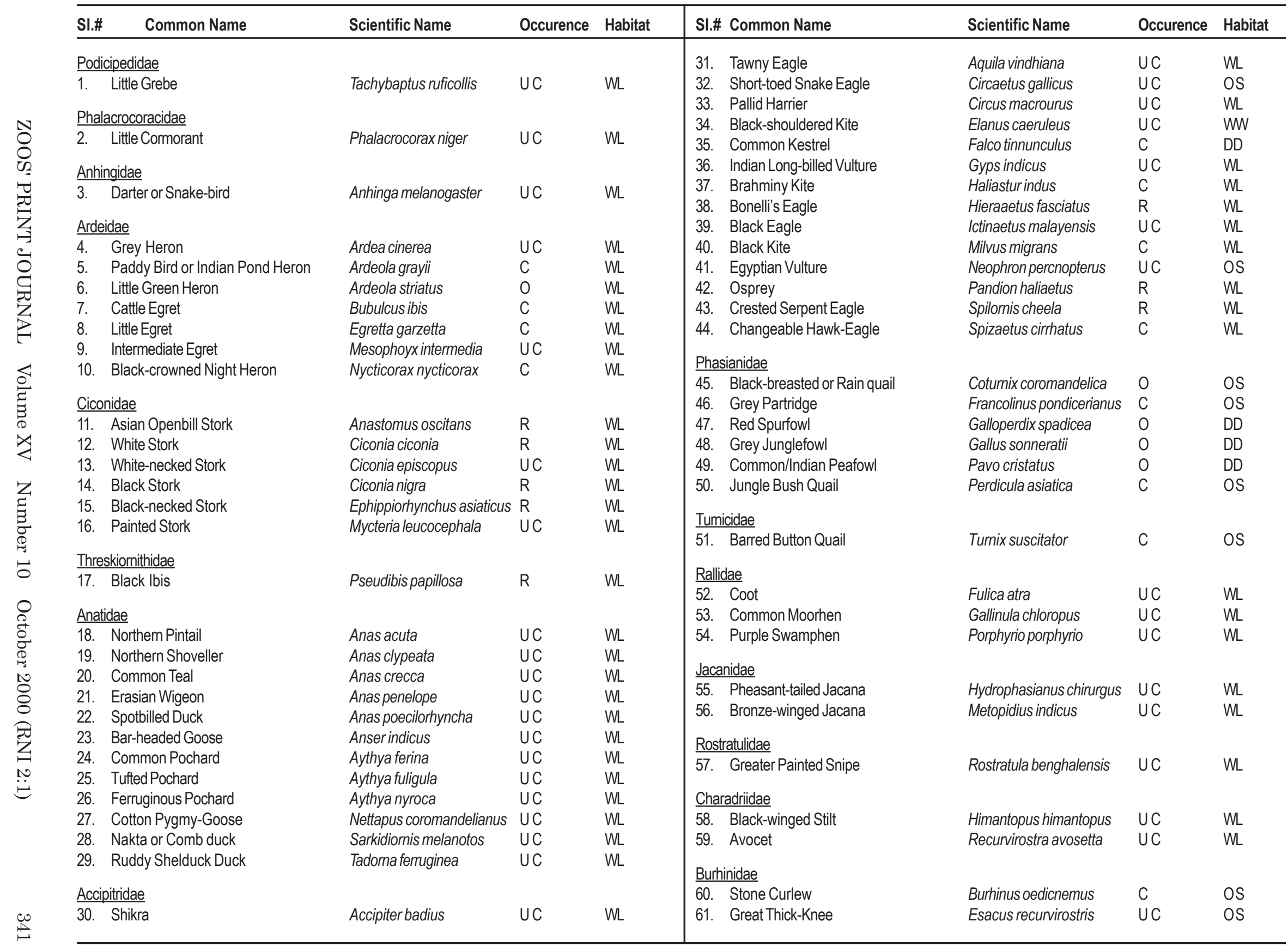




\begin{tabular}{ll}
\hline SI.\# & Common Name \\
\hline Charadridae \\
\hline 62. & Little Ringed Plover \\
63. & Red-wattled Lapwing \\
64. & Yelllow-wattled Lapwing
\end{tabular}

s

Scolopacidae

65. Common Snipe

66. Pintail Snipe

67. Eurasian Curlew

68. Common Sandpiper

69. Green Shank

70. Green Sandpiper

71. Marsh Sandpiper

Pteroclidae

72. Chestnut-belliedSandgrouse

73. Painted Sandgrouse

Columbidae

74. Emerald Dove

75. Blue Rock Pigeon

76. Spotted Dove

77. Eurasian collared Dove

78. Laughing Dove

79. Yellow legged Green Pigeon

Psittacidae

80. Blossom-headed Parakeet

81. Rose-ringed Parakeet

Cuculidae

82. Pied Cuckoo

83. Asian Koel

84. Indian Hawk-Cuckoo

85. Small Blue faced Malkoha

86. Indian Drongo Cuckoo

Centropodidae

87. Greater Coucal

\section{Strigidae}

\section{Spotted Owlet}

89. Eurasian Eagle-Owl

\section{Caprimulgidae}

90. Common Indian Nightjar

91. Grey Nightjar

\begin{tabular}{|c|c|c|}
\hline Scientific Name & Occurence & $\mathrm{Hab}$ \\
\hline Charadrius dubius & 0 & WL \\
\hline Vanellus indicus & C & WL \\
\hline Vanellus malabaricus & C & WL \\
\hline Gallinago gallinago & UC & WL \\
\hline Gallinago stenura & UC & WL \\
\hline Numenius arquata & 0 & WL \\
\hline Tringa hypoleucos & UC & WL \\
\hline Tringa nebularia & UC & WL \\
\hline Tringa ochropus & UC & WL \\
\hline Tringa stagnatilis & UC & WL \\
\hline Pterocles exustus & C & OS \\
\hline Pterocles indicus & 0 & DD \\
\hline Chalcophaps indica & C & DD \\
\hline Columba livia & UC & WW \\
\hline Streptopelia chinensis & C & OS \\
\hline Streptopelia decaocto & C & OS \\
\hline Streptopelia senegalensis & C & OS \\
\hline Treron phoenicoptera & 0 & WW \\
\hline Psittacula cyanocephala & UC & $\mathrm{DD}$ \\
\hline Psittacula krameri & C & $\mathrm{DD}$ \\
\hline Clamatorjacobinus & C & WW \\
\hline Eudynamyus scolopacea & C & $\mathrm{DD}$ \\
\hline Heirococcyx varius & C & DD \\
\hline Phaenicophaeus viridirostris & $\mathrm{C}$ & WW \\
\hline Surniculus lugubris & C & $\mathrm{DD}$ \\
\hline Centropus sinensis & C & $\mathrm{DD}$ \\
\hline Athene brama & C & $\mathrm{DD}$ \\
\hline Bubo bubo & C & WW \\
\hline Caprimulgus asiaticus & C & OS \\
\hline Caprimulgus indicus & C & $\mathrm{DD}$ \\
\hline
\end{tabular}

\section{SI.\# Common Name \\ Apopidae \\ 92. House Swift \\ 93. Palm Swift}

94. Alpine Swift

Hemiprocniodae

95. Crested Tree Swift

Trogonidae

96. Southern Trogon

Cerylidae

97. Pied Kingfisher

Alcedinidae

98. Common Kingfisher

Meropidae

99. Green Bee-eater

100. Blue-tailed Bee-eater

Coraciidae

101. Indian Roller or Blue Jay

Upupidae

102. Common

Bucerotidae

103. Common Grey Hornbill

Megalaimidae

\section{Blue-throated Barbet}

105. Coppersmith Barbet

106. Brown-headed Barbet

Picidae

107. Rufous Woodpecker

108. Yellow-crowned Woodpecker

Scientific Name

Occurence Habitat

Apus affinis

os

Cypsiurus balasiensis C $\quad$ OS

Tachymarptismelba C OS

Hemiprocne coronata $\quad 0 \quad$ DD

Harpactes fasciatus $\quad$ R

Ceryle rudis UC

WL

Alcedo atthis

WL

Merops orientalis $\quad$ C $\quad$ OS

Merops philippinus 0 OS

Coracias benghalensis C OS

Upupa epops $\quad$ UC

DD

Ocyceros birostris UC UW

109. Black-rumped Flamebacked Woodpeckndrocopos maharattensis U C

Celeus brachyurus $\quad$ UC $\quad$ DD

Dendrocopos maharattensis UC OS

Pittidae

110. Indian Pitta

Pitta brachyura UC

OS

Alaudidae

111. Bar-tailed Finch Lark

112. Ashy crowned Finch Lark

113. Rufous-winged Bush Lark

114. Red-winged Bush lark

Ammomanesphoenicurus UC

US OS

Eremopterix grisea UC OS

Mirafra assamica UC OS

Mirafra erythroptera UC OS 


\begin{tabular}{|c|c|c|c|c|c|c|c|}
\hline SI.\# Common Name & Scientific Name & Occurance & Habitat & SI.\# Common Name & Scientific Name & Occurance & Habitat \\
\hline Hirundinidae & & & & 147. Yellow-eyed Babbler & Chrysomma sinense & UC & os \\
\hline 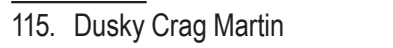 & Hirundo concolor & UC & os & 148. Tawny-bellied Babbler & Dumetia hyperythra & UC & os \\
\hline 116. Streak-throated Swallow & Hirundo fluvicola & C & OS & 149. Common Tailor Bird & Orthotomus sutorius & UC & OS \\
\hline \multirow[t]{2}{*}{ 117. Wire-tailed swallow } & \multirow[t]{2}{*}{ Hirundo smithii } & \multirow[t]{2}{*}{ C } & \multirow[t]{3}{*}{ WW } & 150. Indian Scimitar Babbler & Pomatorhinus horsfieldii & UC & $\mathrm{DD}$ \\
\hline & & & & 151. Lesser Whitethroat & Sylvia curruca & UC & os \\
\hline Lanidae & & & & 152. Orphean Warbler & Sylvia hortensis & UC & OS \\
\hline 118. Brown Shrike & Lanius cristatus & UC & DD & 153. Pale-capped Babbler & Turdoides affinis & UC & os \\
\hline 119. Long-tailed Shrike & Lanius schach & UC & $\mathrm{DD}$ & 154. Common Babbler & Turdoides caudatus & C & OS \\
\hline 120. Bay-backed Shrike & Lanius vittatus & UC & os & 155. Large Grey Babbler & Turdoides malcolmi & UC & OS \\
\hline \multicolumn{4}{|l|}{ Corvidae } & 156. Jungle Babbler & Turdoides striatus & C & os \\
\hline$\overline{\text { 121. lora }}$ & Aegithina tiphia & C & os & \multicolumn{4}{|l|}{ Cisticolodae } \\
\hline 122. Ashy Swallow Shrike & Artamus fuscus & UC & OS & 157. Zitting cisticola & Cisticola juncidis & UC & os \\
\hline 123. Jungle Crow & Convus macrorhynchos & C & OS & 158. Plain Prinia & Prinia inornata & UC & OS \\
\hline 124. House Crow & Corvus splendens & C & os & 159. Ashy Prinia & Prinia socialis & UC & OS \\
\hline 125. Tree Pie & Dendrocitta vagabunda & C & $\mathrm{DD}$ & & & & \\
\hline 126. White-bellied Drongo & Dicrurus caerulescens & C & $\mathrm{DD}$ & Muscicapidae & & & \\
\hline 127. Grey or Ashy Drongo & Dicrurus leucophaeus & UC & WW & 160. Shama & Copsychus malabaricus & UC & $\mathrm{DD}$ \\
\hline 128. Black Drongo or King Crow & Dicrurus macrocercus & C & OS & 161. Magpie-Robin & Copsychus saularis & C & OS \\
\hline 129. Greater Racket-tailed Drongo & Dicrurus paradiseus & UC & WW & 162. Grey-headed Flycatcher & Culicicapa ceylonensis & UC & WW \\
\hline 130. Eurasian Golden Oriole & Oriolus oriolus & UC & WW & 163. Tickell's Blue Flycatcher & Cyornis tickelliae & UC & $\mathrm{DD}$ \\
\hline 131. Black-hooded Oriole & Orilus xanthornus & UC & WW & 164. Pied Bushchat & Saxicola caprata & UC & os \\
\hline 132. Small Minivet & Pericrocotus cinnamomeus & C & OS & 165. Indian Robin & Saxicoloides fulicata & C & OS \\
\hline 133. White-throated Fantail & Rhipidura albicollis & $\mathrm{R}$ & $\mathrm{DD}$ & 166. Indian Black Bird & Turdus merula & UC & WW \\
\hline 134. White-browed Fantail & Rhipidura aureola & $\mathrm{R}$ & $\mathrm{DD}$ & & & & \\
\hline 135. Lesser Wood Shrike & Tephrodornis pondicerianus & $\mathrm{C}$ & $\mathrm{DD}$ & $\underline{\text { Passeriidae }}$ & & & \\
\hline \multirow{2}{*}{ 136. Asian Paradise Flycatcher } & \multirow[t]{3}{*}{ Terpsiphone paradisi } & \multirow[t]{3}{*}{ UC } & \multirow[t]{3}{*}{ WW } & 167. Black-headed Munia & Lonchura malacca & C & WL \\
\hline & & & & 168. Scaly-breasted Munia & Lonchura punctulata & C & os \\
\hline Sturnidae & & & & 169. White-rumped Munia & Lonchura striata & $\mathrm{C}$ & os \\
\hline 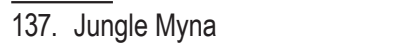 & Acridotheres fuscus & UC & $\mathrm{DD}$ & 170. Grey Wagtail & Motacilla cinerea & UC & WL \\
\hline 138. Common Myna & Acridotheres tristis & C & os & 171. Yellow Wagtail & Motacilla flava & UC & WL \\
\hline \multirow[t]{2}{*}{ 139. Brahminy Starling } & \multirow[t]{3}{*}{ Sturnus pagodarum } & \multirow[t]{3}{*}{$\mathrm{R}$} & \multirow[t]{3}{*}{ OS } & 172. Large Pied Wagtail & Motacilla maderaspatensis & UC & WL \\
\hline & & & & 173. House Sparrow & Passer domesticus & $\mathrm{C}$ & OS \\
\hline Irenidae & & & & 174. Baya Weaver Bird & Ploceus philippinus & $\mathrm{C}$ & WL \\
\hline 140. Gold-fronted Leafbird & Chloropsis aurifrons & $\mathrm{C}$ & DD & & & & \\
\hline \multirow[t]{2}{*}{ 141. Jerdon's Leafbird } & \multirow[t]{3}{*}{ Chloropsis cochinchinensis } & \multirow[t]{3}{*}{ UC } & \multirow[t]{3}{*}{$\mathrm{DD}$} & $\underline{\text { Nectarinidae }}$ & & & \\
\hline & & & & 175. Tickell's Flowerpecker & Dicaeum erythrorhynchos & UC & os \\
\hline Pycnonotidae & & & & 176. Purple Sunbird & Nectarinia asiatica & C & OS \\
\hline 142. Yellow-browed Bulbul & Iole indica & $\mathrm{R}$ & WW & 177 Purnle-rumned Sunbird & Nectarinia zeylonica & UC & os \\
\hline 143. Red-vented Bulbul & Pycnonotus cafer & C & OS & & & & \\
\hline 144. Red-whiskered Bulbul & Pycnonotus jocosus & C & os & Zosteropidae & & & \\
\hline 145. White-browed Bulbul & Pycnonotus luteolus & UC & os & 178. Oriental White-eye & Zosterops palpebrosa & 0 & os \\
\hline \multicolumn{4}{|l|}{ Sylviidae } & & & & \\
\hline 146. Blyth's Reed Warbler & Acrocephalus dumetorum & UC & os & \multicolumn{4}{|c|}{$\begin{array}{l}\text { Occurrence: Common (C), Occassional (O), Uncommon (UC), Rare (R) } \\
\text { Habitat: Well Wooded (WW), Dry Deciduous (DD), Open Scrub (OS), Wet Land (WL). }\end{array}$} \\
\hline
\end{tabular}

